\title{
The impact of restorative audio-visual environment learning on the stress
}

\author{
Nikolai Matveev ${ }^{1}$, Kirill Shamritskiy ${ }^{1}$ \\ ${ }^{1}$ The St.-Petersburg ITMO UNIVERSITY
}

\begin{abstract}
The experiment, conducted in the laser theater Lux Aeterna on the basis of the ITMO University, was aimed at testing the hypothesis about the positive effect of dynamic audio-visual content on the psycho-emotional state of a person and her academic progress after a long period of exposure. Both groups underwent a lengthy Bourdon test and a Shcherbatyh test for the learning stress. Only the participants who passed the laser theater session improved their productivity in the performance of monotonous work in the further passage of the test and showed a significant decrease in the level of general learning stress at the end of the experiment. Correlations between the level of learning stress and academic progress were not found. The use of sessions of dynamic audio-visual content based on the laser theater Lux Aeterna can be considered as an alternative relaxation method that allows changing the level of learning stress in people, increasing productivity in the performance of monotonous work and improve general well-being.
\end{abstract}

\section{Introduction}

Over the last decade a significant number of studies were conducted in order to explore the influence of light on the human body $[1,2,3]$. Static artificial light can influence the psycho-emotional and psycho-physiological state of a person, both in a positive and negative way. [3].

In their everyday life people are exposed to dynamic lighting and changing visual content both in cities (e.g. advertising banners, a flickering lighting of shops and public buildings) and at home (e.g. screens of electronic devices). Daily exposure to such an environment can provoke of rapid fatigue, reduced efficiency, as well as stress and depression. And also it has a detrimental effect on the physiological health [1]. To reduce the negative impact of these factors, there are various techniques used both in medicine and in everyday life. Visual content is an example of one of the instruments of influence. It can be divided into two types: static and dynamic visual content. Each type has its own nature and the impact on the work of our perception organs and the analysis of environmental information. Various studies worldwide explore various kinds of visual content and its impacts on human beings.

The beneficial effects on the level of mental fatigue were proven for "restorative environments", such as natural landscapes [4]. Psychologists in relaxation therapy used audio content to accelerate the rehabilitation in cases of depression or reduce a general level of stress [5].

However, not only visual content that we observe in the natural environment can be used to improve the psycho-emotional state of a person. It is proven that the observation of a complex visual structure based on the selfsimilarity of elements (i.e. fractal images) activates the areas of the human brain responsible for relaxation and recovery processes, and also improves the functional state of the brain $[6,7]$. This should take into account the characteristics responsible for the reproduction and quality of visual content. Images with a frame rate of $10 \mathrm{~Hz}$ activate the nerve endings of the brain better than blurred or Quick-images. [8]

The effects of the dynamic visual content used in the performances of the laser theater Lux Aeterna are similar to the techniques described above. A group of scientific researchers, who aimed at studying and analyzing the impact of visual content on the human condition created by using laser radiation, was created on the basis of ITMO University [9-11]. Previous experiments were conducted only using the pre-/ post-test method, so we want to determine the effect for long-term use.

A new research study was conducted to assess the impact of sessions performed in the laser theater Lux Aeterna on a larger population sample, and regularly observe and evaluate the impact of the dynamic visual content generated by the theater on the psycho-emotional state of people.

\footnotetext{
${ }^{1}$ E-mail adress: matveev_nv@mail.ru , ikirill2193@gmail.com
} 


\section{Methods}

\subsection{Participants}

In total, 70 students enrolled in the first year of the bachelor's degree at the ITMO University initially participated in the study; however, 30 participants ( 20 female and 10 male, aged $20 \pm 3$ years) were admitted to data processing due to the irregular participation in the group of participants. Each participant gave informed consent to participate in the study by filling out the form in accordance with ICH Harmonised Tripartite Guideline for GCP. To distribute the participants across equal groups, testing was conducted to determine the level of educational stress. Based on the data received, the participants were divided into two equally sized groups: the work group and the control group. The composition of the groups took into account the data on student achievement in the first semester of studies and the factors that potentially affect the level of learning stress among students (e.g. residence at the time of the experiment: with parents / in the dormitory / in the apartment (separately)), general emotional state, and subjective evaluation of a personal stress level.

\subsection{Procedure}

The premises of the laser theater Lux Aeterna operating on the basis of the ITMO University were selected as the site of the experiment, where the visual content was broadcasted. The theater is a spherical dome (planetarium) with the height of $6.3 \mathrm{~m}$ and the diameter of $10 \mathrm{~m}$. The number of seats is 30 . The dynamic visual content was generated using laser radiation (Kvant laser module / $3 \mathrm{~W} /$ full color equipment). The results of recent studies, conducted in the dome of the laser theater, showed a positive effect of visual content on human performance and on brain function (states of altered consciousness [12], relaxation). In this regard, it was decided to assess the influence of visual content generated by laser radiation on the psycho-emotional state of a person. The dynamic visual content is projected on the screen placed under the spherical dome of the laser theater by passing the laser radiation through the liquid optical medium and optical elements arranged on a movable platform. As the laser is reflected and refracted in the optical media, the display content, consisting of complex shapes with a high degree of self-similarity, can be observed. To assess the degree of self-similarity, the fractal dimension of the image is used, which helps create unique (randomly generated) content and obtain visual images based on the optical characteristics and mathematical calculations. The obtained images projected on the spherical screen of the theater are similar to their natural analogs. They have smooth shapes and outlines that can be compared to the examples of a natural landscape or represented in the form of some kind of living organisms. Visual content with the elements of natural similarity does not cause discomfort to the viewer, as it is subconsciously perceived by her as a natural image. At the same time, the variable dynamic content is achieved by using various combinations of optical elements. The visual content session is accompanied by a calm audio composition.

During the experiment, the students in the work and control groups were required to pass a Bourdon test once every two weeks, a proof-of-effect test to assess the volume, concentration and sustainability of attention. The test is conducted by means of special forms with rows of random (Cyrillic alphabet) letters. The participant viewing the form row by row selects the letters indicated in the instruction by clicking computer mouse buttons. The test run time is 10 minutes; every 60 seconds the algorithm for selecting the character in the line is automatically changed. Thus, the participant of the test needs to monitor the correctness and the way of selecting the symbols. After all data measurements were made, the work group was proposed to assist a laser performance session of 20-25 min in the theater dome. This series of procedures, organized during the whole spring semester, was required for the participants to pass the test.

To determine the level of their educational stress, the students were asked to take the test based on the method of Yu.V.Scherbatyh. The testing was carried out at the beginning and in the end of the experiment. A psychological test included a set of questions divided into three parts: the contribution of individual factors to the overall state of stress (14 questions); the stress associated with the study (18 questions), and the stress management techniques (4 questions). Each part included several questions, assessing its response from 1 to 10 points. For the part with the multiple choice, it was required to note the choice made by the student, or make own choice if there was no suitable option. The total average score reflecting the overall level of participant's learning stress (from 1 to 10 points) was calculated based on the results of the answers obtained for three parts.

\section{Results}

At the end of the experiment, we obtained the output from the Bourdon test results and the Shcherbatykh test, helping determine the level of educational stress and academic performance of the participating students in the last two semesters. The results of the output parameters obtained from the Burdon test- the correctness factor and the 
productivity factor $(\mathrm{PF})$ - were analyzed. The correctness factor was calculated as the ratio of the total volume of relatively correctly scanned symbols and erroneously (or missed) selected values. The PF was calculated as the multiplication of the correctness factor by the total number of scanned symbols. The dynamic changes in the mean values of the PFs calculated for the work group and the control group is shown in the graph below (Fig. 1). On both graphs, the confidence intervals are plotted for the PF values. The scores corresponding to the productivity factor are marked on the scale Y.

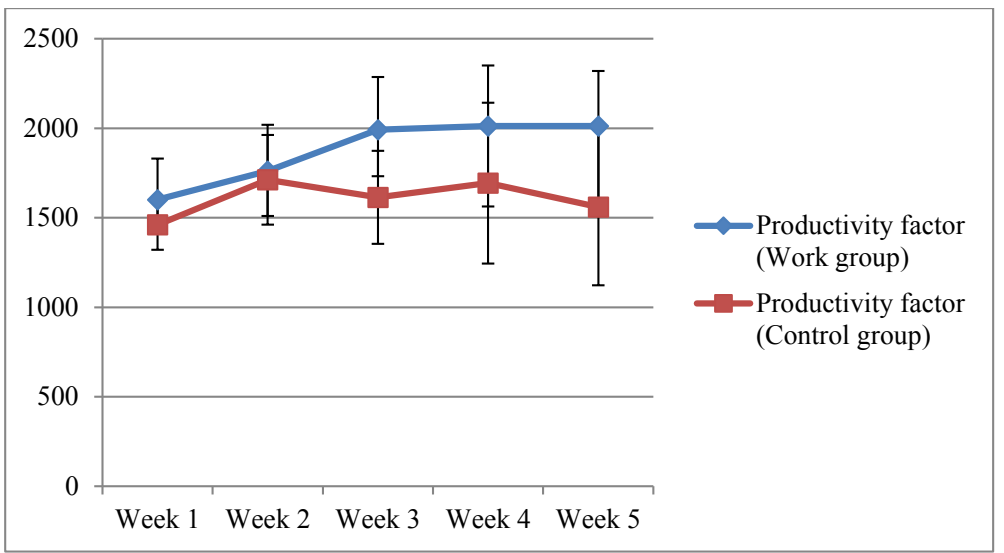

Fig. 1. The change in the productivity factor (mean value) during the experiment.

Having obtained the PF values, we analyzed two related data samples by calculating the Wilcoxon signedrank test for the work and control groups in the beginning and at the end of the experiment. The criterion for Wilcoxon signed-ranks allows us to determine whether the change in the PF (and the passing of the Bourdon test as a whole) affects the viewing of visual content during the experiment. The Wilcoxon signed-rank test is intended to compare the values measured under two different conditions on the same sample of subjects. It allows us to establish not only the direction of changes but also their severity, that is, it is able to determine whether the shift in one direction is more intensive than in the other. The values for the first and the fifth week of the experiment were compared. In order to draw conclusions about the effectiveness of the impact of visual content on the test Bourdon results for the work and control groups, the statistical significance asymptotic value must be less than 0.05 . Statistics $\mathrm{Z}$ shows the standard deviation of values between the first and the fifth week. Test statistic for the work group is $Z=2,4$ (in absolute value); two-sided asymptotic significance of 0.016 . For the control group test statistic is $Z=0.7$; two-sided asymptotic significance 0.448 . Since the asymptotic significance for the work group is less than 0.05 , the $\mathrm{Z}$ statistics show that the presence of visual content effectively affects the stability of the productive passage of the Bourdon test and the improvement of the final totals. In the control group, the asymptotic significance is greater than 0.05 , which means that in the absence of visual content during the experiment, the results of the Bourdon test do not have any significant changes at the beginning and end points of the study, or the lack of positive dynamics in the change in the parameter of the PF. Table 1 presents the results of statistical criteria for the Wilcoxon signed-rank test for all of the conducted methodologies (Table 1).

Table 1. Statistical criteria the Wilcoxon test according to test results

\begin{tabular}{|c|c|c|c|c|c|c|}
\hline Statistical & & Work & & & Contr & up \\
\hline Tests criteria & $\begin{array}{c}\Delta \text { mean } \\
\quad(5 \\
\text { week- } \\
1 \text { week) }\end{array}$ & Z & $\begin{array}{l}\text { Asymptotic } \\
\text { significance }\end{array}$ & $\begin{array}{c}\Delta \text { mean } \\
\quad(5 \\
\text { week- } \\
\text { 1week) }\end{array}$ & Z & $\begin{array}{l}\text { Asymptotic } \\
\text { significance }\end{array}$ \\
\hline $\begin{array}{l}\text { Bourdon test } \\
\text { (PF points) }\end{array}$ & 411 & 2.401 & 0.016 & 86.6 & 0.7 & 0.484 \\
\hline $\begin{array}{c}\text { Shcherbatyh } \\
\text { Test }\end{array}$ & -1.3 & 2.521 & 0.012 & 0.8 & 1.897 & 0.058 \\
\hline
\end{tabular}




\begin{tabular}{|c|c|c|c|c|c|c|}
\hline $\begin{array}{c}\text { Academic } \\
\text { progress }\end{array}$ & 0.2 & 1.265 & 0.206 & 0.2 & 0.541 & 0.589 \\
\hline
\end{tabular}

Based on the results of the Shcherbatyh learning stress test, we obtained several data samples with the values of the general level of learning stress in the subjects at the time of the experiment and after its completion. The analysis of the test results of the work group showed a significant reduction in the level of learning stress in $80 \%$ of the group (a decrease of -1.3 by $0.3-1.3$ points). This indicator did not change in $15 \%$, and in $5 \%$ of the work group, the level of learning stress increased (an increase of -1.3 by $0.1-0.4$ points). After calculating the Wilcoxon signed-rank criterion for the values of the learning stress level (before and after the experiment), the values of $Z=2.521$ and the asymptotic significance (bilateral) of 0.012 were obtained. Due to the fact that the asymptotic significance is less than 0.05 , the data can be considered reliable, and the $\mathrm{Z}$ statistics indicate a significant change in the level of learning stress. In the case of the control group, the results showed that $66 \%$ of the subjects had an increase in the level of total stress (an increase of 0.8 by $1-3.3$ points), $30 \%$ of participants decreased their level of total stress (a decrease of 0.8 by $0.1-0.7$ points), and for $4 \%$,it remained unchanged. In the calculation of the criterion for the signed-ranks of Wilcoxon, the values $Z=1.897$ and the asymptotic significance of 0.058 were obtained. The last indicator is more than 0.05 , which means that the control group changes in the level of learning stress are insignificant and depend exclusively on the subjective characteristics of the subjects in the control group.

In conclusion, the analysis of the results of academic progress among the students of the work and control groups, namely the assessments for two semesters in the exact disciplines (physics, mathematics) were carried out. The exact disciplines were chosen as a factor for analysis, as these disciplines require increased concentration of attention during the students' learning process. The results of the average score for the six months in these disciplines did not show any significant change. After calculating the criterion for Wilcoxon's signed-ranks, we obtained the following statistics: for the work group, $Z=1.265$ and asymptotic significance 0.206 ; in the control group, $Z=0.541$ and asymptotic significance 0.589 . The asymptotic significance indicators are greater than 0.05 in both groups, which points to either a slight change in the academic progress in the exact disciplines or to a complete absence of such changes.

\section{Discussion}

The research was aimed at assessing the impact of dynamic audiovisual content on a person's psychoemotional state, subject to regular visits to sessions based on the performances of the laser theater Lux Aeterna. To this end, three sets of data were tested: the productivity and sustainability of attention, the general level of learning stress and academic progress of students. Based on the results obtained, it can be concluded that regular attendance of audiovisual content sessions using the laser theater Lux Aeterna can have a positive impact on productivity and concentration of attention in the performance of monotonous work (with the presence of emotional and intellectual load) or help maintain stable results in the performance of learner. Also, the regular exposure to dynamic audiovisual content can significantly reduce the level of learning stress. The research showed a decrease in the level of learning stress by more than three points from the initial level among $80 \%$ of the subjects. In addition, at the end of the test, a survey was conducted among those for whom the test shows a decrease in the level of stress, and in $60 \%$ of cases, the results of self-reported confirmed the results of the Shcherbatykh test. Correlations between the level of learning stress and academic progress were not found. Thus, it can be argued that there is no influence of dynamic audiovisual content on the student's progress and this parameter depends only on the subjective features of each participant in the experiment.

In this regard, the use of dynamic audio-visual content sessions based on the laser theater Lux Aeterna can be considered as an alternative relaxation method that allows changing the level of learning stress in people, increasing productivity in the performance of monotonous work and improving general well-being. The key factor for obtaining a positive result will be the regular use of this technique in the long term.

The researched content can be offered in specially allocated restorative rooms. However, the specific features of working with the visual image of the Lux Aeterna theater can be applied when creating general lighting in working and home environments. Studying the dynamic ranges of brightness and radiation spectrum variation can help identify the patterns that positively influence the psychoemotional state of a person, and transfer them to the operating modes of the common lighting systems. 


\section{References}

1. Ioffe K.I. "Biological influence of visible light on the human body." International Scientific and Technical Journal "Lighting Engineering and Power Engineering. No. 3 (2008): 21-29.

2. Ovchinnikov S.S. and Serobaba A.A. "Evaluation of the effectiveness of the influence of the light environment on the human body." Lighting and electric power industry. No. 4 (2008): 4-10.

3. Zakgeim, Alexander L. "Light-emitting Diode Illumination Systems: Energy Efficiency, Visual Perception, and Safety for Health." Light \& engineering 21.2 (2013).

4. Berto, Rita. "Exposure to restorative environments helps restore attentional capacity." Journal of environmental psychology 25.3 (2005): 249-259.

5. Beck, Bolette Daniels, Åse Marie Hansen, and Christian Gold. "Coping with work-related stress through Guided Imagery and Music (GIM): Randomized controlled trial." Journal of music therapy 52.3 (2015): 323-352.

6. Tkacheva, L. O. "Effects of fractal dynamic images on human functional state." Vestnik Saint-Petersburg University (RUS). 12.2 (2010).

7. Tkacheva, L. O., I. A. Gorbunov, and A. D. Nasledov. "Reorganization of system brain activity while understanding visually presented texts with the increasing completeness of information." Human Physiology 41.1 (2015): 11-21.

8. Bakardjian, Hovagim, Toshihisa Tanaka, and Andrzej Cichocki. "Emotional faces boost up steady-state visual responsesforbrain-computer interface." Neuroreport 22.3 (2011): 121-125.

9. Matveev N., Prokopenko V., Sapunova N., Fridman D., Research into the influence of light-music performances on psychophysiological states, Light \& Engineering - 2016, Vol. 24, No. 2, pp. 22-24.

10. Matveev N., Shcheglov S., Romanova G., Koneva T., Modeling of the laser device for the stress therapy// Proceedings of SPIE - 2017, Vol. 10239, pp. 102390H

11. Morgia S. Wellness keeps aging workforce young. Benefits and pensions monitor, 2014, 24, pp 36-37.

12. Ludwig A. "Altered states of consciousness" Archives of general psychiatry. — N 15, 1966. P. 225-234. 